\title{
Dibutyryl cyclic AMP decreases capacitation time in vitro in mouse spermatozoa
}

\author{
Lynn R. Fraser \\ Department of Human Biology, Chelsea College, Manresa Road, London SW3 6LX, U.K.
}

\begin{abstract}
Summary. Epididymal mouse sperm suspensions were preincubated for various times in medium containing glucose and/or dibutyryl cyclic AMP and then assessed for fertilizing ability in vitro, loss of the acrosome and motility changes. Capacitation time was significantly reduced by exposure to glucose and $0.1 \mathrm{~mm}$-dbcAMP for 30 min as evidenced by early and synchronous fertilization of eggs, compared with glucose alone. Although this was accompanied by a precocious development of whiplash motility, the rate of acrosome loss in isolated sperm suspensions was not accelerated by the presence of exogenous cyclic nucleotide. Exposure of spermatozoa to $1 \mathrm{mM}$-dbcAMP in the presence of glucose resulted in very poor fertilization, but the effect could be prevented by withholding glucose until eggs were introduced; this may be due to free butyrate in the system since the inclusion of $1 \mathrm{~mm}$-butyrate in glucose-containing medium had a similar inhibitory effect. Although cyclic nucleotide supported the acrosome reaction but not motility changes, no fertilization was obtained unless zonae were removed, when a low level of fertilization (30\%) was observed. Both whiplash motility and acrosome loss are thus obligatory for sperm penetration of the zona and glycolytic metabolism supports both changes, perhaps by promoting endogenous generation of cyclic AMP to act as an intermediary in these two distinct phenomena.
\end{abstract}

\section{Introduction}

The capacitation process confers on mammalian spermatozoa the ability to fertilize eggs, specifically by permitting them to undergo the acrosome reaction which releases enzymes required for penetration of the investments surrounding the unfertilized egg (Bedford, 1970; Morton, 1976). Because the acrosome reaction can be assessed by light microscopy, this characteristic is frequently used as an indicator of capacitation and hence fertilizing ability; another easily detectable change associated with fertilizing ability is the development of the characteristic whiplash pattern of motility by the spermatozoa of some species (Yanagimachi, 1970; Yanagimachi \& Usui, 1974; Fraser, 1977). Amongst the factors implicated in the process of capacitation is cyclic AMP. Caffeine and other methylxanthines, which can act as cyclic nucleotide phosphodiesterase inhibitors, have been observed to increase and even prolong sperm motility (Garbers, First, Sullivan \& Lardy, 1971; Garbers, Lust, First \& Lardy, 1971; Hicks, Martinez-Manautou, Pedron \& Rosado, 1972; Hoskins, 1973; Schoenfeld, Amelar \& Dubin, 1975). While these results do not necessarily indicate a positive effect on fertilizing ability, other studies using in-vitro fertilization systems have suggested that exogenous cyclic AMP supplied as the dibutyryl analogue could play a positive role in fertilization, shortening the time required for capacitation (rat: Toyoda \& Chang, 1974; rabbit: Rosado, Hicks, Reyes \& Blanco, 1974). More 
conclusive evidence of an effect on capacitation has been presented by Fraser (1979b) who demonstrated that caffeine significantly decreased the capacitation time for mouse spermatozoa, promoting rapid penetration of eggs and precocious development of whiplash motility. However, Rogers \& Garcia (1979) have reported that exogenous dibutyryl cyclic AMP and phosphodiesterase inhibitors inhibited both induction of the acrosome reaction and fertilization in vitro by hamster and guinea-pig spermatozoa, results at variance with other published studies which suggested a positive influence for the cyclic nucleotide. In the present study the temporal relationships of achievement of fertilizing ability, acrosome loss and whiplash motility were examined in sperm suspensions incubated in media containing glucose and/or dibutyryl cyclic AMP to determine the possible role played by this cyclic nucleotide in fertilization by mouse spermatozoa.

\section{Materials and Methods}

\section{In-vitro fertilization}

The modified Tyrode's solution described by Fraser \& Drury (1975), containing $5 \cdot 56$ mM-glucose and excluding pyruvate and lactate, was used for all experimental procedures; glucose was also omitted as indicated. Osmolarity was maintained by the addition of extra $\mathrm{NaCl}$ and media contained $30 \mathrm{mg}$ bovine serum albumin (BSA: Armour; Eastbourne, Sussex) per ml unless otherwise specified. Gamete incubations were carried out in $30 \mathrm{~mm}$ plastic tissue-culture dishes (Sterilin) in medium overlaid with equilibrated liquid paraffin (Boots). The gas phase used throughout was $5 \% \mathrm{O}_{2}, 5 \% \mathrm{CO}_{2}, 90 \% \mathrm{~N}_{2}$.

Unfertilized eggs were obtained from outbred TO females induced to superovulate by an i.p. injection of 7.5 i.u. PMSG (Gestyl: Organon, Morden, Surrey) followed approximately $48 \mathrm{~h}$ later by 5 i.u. hCG (Pregnyl: Organon). Females were killed $14 \mathrm{~h}$ after the hCG injection and unless otherwise stated, eggs were released from oviducts directly into sperm suspensions.

When zona-free eggs were required, the following procedure was used, with the basic medium in all steps lacking glucose but containing $4 \mathrm{mg} \mathrm{BSA} / \mathrm{ml}$. Cumulus cells were removed by releasing unfertilized eggs into hyaluronidase-containing medium (300 units $/ \mathrm{ml}$; bovine testes Type 1: Sigma, St Louis, Missouri). Eggs were washed once and then transferred in small groups to a pronase solution ( $0.04 \%$ pronase: Koch-Light, Colnbrook, Bucks). A stock solution of $2 \%$ pronase was prepared in phosphate-buffered saline (PBS), pH 7.4, dialysed overnight against PBS and Millipore-filtered; just before use, this stock was diluted with the basic medium to give the final concentration. After 15-20 sec in the pronase, zonae were much weakened and eggs were transferred to pronase-free medium where zona removal, if not effected, was completed by teasing with a needle. Denuded eggs were transferred to a second aliquot of basic medium and retained there until transfer to the appropriate sperm suspensions.

Epididymal TO sperm suspensions were prepared by releasing the contents of 2 epididymides, 1 from each of 2 males, into $1 \mathrm{ml}$ medium. After a preincubation of $30 \mathrm{~min}$ or $2 \mathrm{~h}$ at $37^{\circ} \mathrm{C}$, suspensions were diluted 10 -fold in the medium used for fertilization to give a final concentration of $1-2 \times 10^{6} \mathrm{spermatozoa} / \mathrm{ml}$ and eggs were added. In most experiments the same medium was used for preincubation and fertilization.

In Series I experiments, sperm suspensions were preincubated for 30 min before addition of eggs; 4 different media combinations were examined. In 3 of these the preincubation and fertilization stages utilized the same medium: (1) glucose only, which served as the control medium; (2) glucose plus 0.1 mM-dibutyryl cyclic AMP (dbcAMP: $\mathrm{N}^{6}, \mathrm{O}^{2 \prime}$-dibutyryladenosine $3^{\prime}: 5^{\prime}$ cyclic monophosphoric acid: Sigma); (3) glucose plus 1 mM-dbcAMP. In the fourth group, the preincubation medium was glucose-free but contained $1 \mathrm{mM}$-dbcAMP, while the fertilization medium contained glucose and $1 \mathrm{~mm}$-dbcAMP. In Series II, these same 4 combinations were tested after preincubation for $2 \mathrm{~h}$. In Series III, sperm fertilizing ability after a $2 \mathrm{~h}$ preincubation 
was tested with zona-free eggs. The 3 media used in this series were: (1) glucose-containing medium; (2) glucose-free medium plus $0.1 \mathrm{mM}$-dbcAMP; (3) preincubation in medium as for (2) and dilution in glucose-containing medium for fertilization.

\section{Assessment}

Fertilization and nuclear development. In all experiments, eggs were transferred from the sperm suspensions into small droplets of glucose-free medium under liquid paraffin approximately $65 \mathrm{~min}$ after gamete mixing. At $75 \mathrm{~min}$, all groups of eggs were fixed simultaneously by flooding the dishes with neutral buffered formalin ( $4 \%$ formaldehyde, final concentration). Eggs were subsequently stained with $0.5 \%$ aceto-orcein and examined microscopically (Fraser, 1979a). An egg was considered to be fertilized if it had resumed meiosis II (activation) and possessed a decondensing sperm head. These two independent characteristics can be further categorized into egg activation stages of early anaphase (chromosome separation just beginning), middle to late anaphase (chromosome separation advanced to mid-spindle position or further) and telophase-second polar body (chromosome separation concluded and extrusion of polar body either begun or completed), and into sperm decondensation stages of (1) just beginning, (2) half-decondensed, (3) fully decondensed. For statistical analysis, $\chi^{2}$ determinations were made.

Acrosome loss. The sperm suspensions used for fertilization in vitro were also examined at 5 $\mathrm{min}, 30 \mathrm{~min}, 2 \mathrm{~h}$ and sometimes $4 \mathrm{~h}$ during incubation in various media for assessment of the acrosome loss which occurred in the absence of eggs. Glucose-free medium was also examined after $2 \mathrm{~h}$ for comparison. All sperm suspensions contain a certain proportion of non-motile, possibly dead cells, and on the assumption that only motile spermatozoa are capable of fertilizing eggs, suspensions were enriched for motile cells prior to assessment. Short column beds $(2 \cdot 5 \mathrm{~cm})$ of Sephadex G-25 were prepared in Pasteur pipettes. Several drops of each concentrated sperm suspension were applied and eluted with the appropriate medium; the filtrate thus obtained had approximately $90 \%$ motile gametes. A drop of each suspension was applied to a clean slide, a small amount of $0.01 \%$ acridine orange was added and a coverslip applied. Since the acridine orange is used for its ability to immobilize the cells rather than for its fluorescent properties, the spermatozoa can then be examined with ordinary bright light for the presence or absence of an acrosome; alternatively, the filtered suspensions can be fixed in buffered formalin prior to assessment. At least 100 spermatozoa were assessed in each sample; for data presented in Table 5 more than 400 cells were examined in each group.

Motility patterns. In all the media tested spermatozoa were examined for the whiplash motility pattern associated with fertilizing ability in the mouse (Fraser, 1977), and the relative proportions of single, motile gametes displaying the characteristic pattern were estimated; again, assessment was made on suspensions in the absence of eggs.

\section{Results}

\section{Series I: sperm preincubation for $30 \mathrm{~min}$}

Spermatozoa released and preincubated for $30 \mathrm{~min}$ in glucose-containing (control) medium were able to fertilize $60.8 \%$ of eggs as judged by the criteria detailed above, and the spread of egg activation and sperm-head decondensation stages indicated asynchronous penetration of these eggs (Table 1). When the test medium contained both glucose and $1 \mathrm{mM}$-dbcAMP, significantly fewer eggs were fertilized $\left(21.4 \% ; \chi^{2}, P<0.001\right)$ and asynchronous penetration was again indicated. In contrast, when glucose was omitted from the preincubation medium containing the dbcAMP and only introduced at the time eggs were added, fertilization rates were much higher $(84.2 \% ; P<0.01)$ and terminal stages of nuclear development were obtained in the majority of eggs, suggesting early and relatively synchronous penetration by the fertilizing spermatozoa. 
When the test medium contained glucose $+0.1 \mathrm{~mm}$-dbcAMP, very high levels of fertilization were obtained $(90.5 \% ; P<0.001)$ with terminal stages of early nuclear development observed in most of the eggs. The lower concentration of dbcAMP therefore enhanced the fertilizing ability of spermatozoa and promoted rapid penetration, compared with medium containing only glucose.

Table 1. Fertilizing ability of mouse sperm suspensions preincubated for $30 \mathrm{~min}$ in media containing $5.56 \mathrm{~mm}$-glucose $(\mathrm{G})$ and/or dibutyryl cyclic AMP (dbcAMP)

\begin{tabular}{|c|c|c|c|c|c|c|c|c|c|}
\hline \multicolumn{2}{|c|}{ Medium for } & \multirow{2}{*}{$\begin{array}{l}\text { No. } \\
\text { of } \\
\text { exps }\end{array}$} & \multirow[b]{2}{*}{ Fertilized } & \multicolumn{3}{|c|}{ Egg stage, \%* } & \multicolumn{3}{|c|}{ Sperm stage, \%* } \\
\hline Preincubation & Fertilization & & & EA & $\mathrm{M}-\mathrm{LA}$ & T-PB2 & 1 & 2 & 3 \\
\hline G & G & 9 & $\begin{array}{c}93 / 153 \\
(60 \cdot 8 \%)\end{array}$ & $24 \cdot 7$ & $32 \cdot 3$ & $43 \cdot 0$ & $60 \cdot 2$ & $11 \cdot 8$ & $28 \cdot 0$ \\
\hline $\begin{array}{c}G+d b c A M P \\
(0.1 \mathrm{mM})\end{array}$ & $\begin{array}{c}G+\text { dbcAMP } \\
(0.1 \mathrm{~mm})\end{array}$ & 2 & $\begin{array}{l}38 / 42 \\
(90.5 \%)\end{array}$ & 0 & $21 \cdot 1$ & 78.9 & $10 \cdot 5$ & $21 \cdot 1$ & $68 \cdot 4$ \\
\hline $\begin{array}{c}G+\text { dbcAMP } \\
(1 \mathrm{~mm})\end{array}$ & $\begin{array}{c}G+\text { dbcAMP } \\
(1 \mathrm{mM})\end{array}$ & 4 & $\begin{array}{c}9 / 42 \\
(21.4 \%)\end{array}$ & $55 \cdot 6$ & $22 \cdot 2$ & $22 \cdot 2$ & $77 \cdot 8$ & $11 \cdot 1$ & $11 \cdot 1$ \\
\hline $\begin{array}{c}\text { dbcAMP } \\
(1 \mathrm{mM})\end{array}$ & $\begin{array}{c}G+\text { dbcAMP } \\
(1 \mathrm{~mm})\end{array}$ & 3 & $\begin{array}{l}32 / 38 \\
(84 \cdot 2 \%)\end{array}$ & $6 \cdot 3$ & $18 \cdot 8$ & $75 \cdot 0$ & $31 \cdot 3$ & $18 \cdot 8$ & $50 \cdot 0$ \\
\hline
\end{tabular}

EA, early anaphase; M-LA, middle-late anaphase; T-PB2, telophase-2nd polar body.

* Eggs were fixed after $75 \mathrm{~min}$.

\section{Series II: sperm preincubation for $2 h$}

When mouse sperm suspensions are preincubated for $2 \mathrm{~h}$ in the control medium, previous studies have shown that there is rapid, synchronous penetration by spermatozoa, resulting in high levels of fertilization and terminal stages of egg activation and sperm head decondensation (Fraser, 1979a, b; Fraser \& Quinn, 1981). The present results were entirely consistent with these observations. Of the 4 combinations of media tested, 3 yielded $>95 \%$ fertilization with approximately $90 \%$ or more of eggs containing terminal stages of nuclear development (Table 2). As in Series I, medium containing glucose $+1 \mathrm{mM}$-dbcAMP throughout the experimental period supported only low levels of fertilization $(22.2 \% ; P<0.001)$ with a spread in nuclear stages which suggested asynchronous penetration over a relatively long time. The possibility that this

Table 2. Fertilizing ability of mouse sperm suspensions preincubated for $2 \mathrm{~h}$ in media containing $5.56 \mathrm{mM}$-glucose $(\mathrm{G})$ and/or dibutyryl cyclic AMP (dbcAMP)

\begin{tabular}{|c|c|c|c|c|c|c|c|c|c|}
\hline \multicolumn{2}{|c|}{ Medium for } & \multirow{2}{*}{$\begin{array}{l}\text { No. } \\
\text { of } \\
\text { exps }\end{array}$} & \multirow[b]{2}{*}{ Fertilized } & \multicolumn{3}{|c|}{ Egg stage, \%* } & \multicolumn{3}{|c|}{ Sperm stage, \%* } \\
\hline Preincubation & Fertilization & & & EA & M-LA & $\mathrm{T}-\mathrm{PB} 2$ & 1 & 2 & 3 \\
\hline $\mathrm{G}$ & G & 9 & $\begin{array}{c}94 / 95 \\
(98 \cdot 9 \%)\end{array}$ & 0 & $2 \cdot 1$ & 97.9 & $3 \cdot 2$ & $6 \cdot 4$ & 90.4 \\
\hline $\begin{array}{c}\mathrm{G}+\text { dbcAMP } \\
(0.1 \mathrm{mM})\end{array}$ & $\begin{array}{c}G+\text { dbcAMP } \\
(0.1 \mathrm{mM})\end{array}$ & 2 & $\begin{array}{c}25 / 25 \\
(100 \%)\end{array}$ & 0 & 0 & 100 & 0 & 0 & 100 \\
\hline $\begin{array}{c}G+\text { dbcAMP } \\
(1 \mathrm{mM})\end{array}$ & $\begin{array}{c}\mathrm{G}+\mathrm{dbcAMP} \\
(1 \mathrm{mM})\end{array}$ & 4 & $\begin{array}{c}6 / 27 \\
(22 \cdot 2 \%)\end{array}$ & $16 \cdot 7$ & $33 \cdot 3$ & $50 \cdot 0$ & $33 \cdot 3$ & $16 \cdot 7$ & 50.0 \\
\hline $\begin{array}{c}\text { dbcAMP } \\
(1 \mathrm{mM})\end{array}$ & $\begin{array}{c}G+\text { dbcAMP } \\
(1 \mathrm{~mm})\end{array}$ & 3 & $\begin{array}{c}26 / 27 \\
(96 \cdot 3 \%)\end{array}$ & 0 & 0 & 100 & 0 & $11 \cdot 5$ & 88.5 \\
\hline $\begin{array}{c}\mathrm{G}+\text { butyrate } \\
(1 \mathrm{mM})\end{array}$ & $\begin{array}{c}G+\text { butyrate } \\
(1 \mathrm{~mm})\end{array}$ & 2 & $\begin{array}{c}10 / 30 \\
(33 \cdot 3 \%)\end{array}$ & 0 & $60 \cdot 0$ & $40 \cdot 0$ & $20 \cdot 0$ & $10 \cdot 0$ & $70 \cdot 0$ \\
\hline $\begin{array}{c}\mathrm{G}+\text { butyrate } \\
(0.1 \mathrm{mM})\end{array}$ & $\begin{array}{c}\mathrm{G}+\text { butyrate } \\
(0.1 \mathrm{mM})\end{array}$ & 2 & $\begin{array}{c}26 / 30 \\
(86 \cdot 7 \%)\end{array}$ & 0 & 3.9 & $96 \cdot 1$ & 0 & $3 \cdot 9$ & $96 \cdot 1$ \\
\hline
\end{tabular}

EA, early anaphase; M-LA, middle-late anaphase; T-PB2, telophase-2nd polar body.

* Eggs were fixed after $75 \mathrm{~min}$. 
observed inhibition might be due to relatively high levels of butyrate, present both in the initial cyclic nucleotide sample and additional butyrate released during metabolism of the cyclic nucleotide, or adenosine was examined. Results obtained with medium containing glucose + butyrate indicated that butyrate at a concentration of $1 \mathrm{~mm}$ did interfere with fertilization, leading to low levels of fertilization (33.3\%) and a spread of nuclear stages similar to those observed with 1 mM-dbcAMP (Table 2). Adenosine ( $1 \mathrm{~mm}$ ) was also examined but did not mimic the inhibition observed with butyrate (data not shown).

\section{Series III: fertilization of zona-free eggs}

In the presence of glucose and cyclic nucleotide, spermatozoa underwent the acrosome reaction and developed whiplash motility, while in the presence of cyclic nucleotide alone, acrosome loss was observed without change in the motility pattern (discussed below). Because whiplash motility may be important in zona penetration, zona-intact and zona-free eggs were used to test the fertilizing ability of spermatozoa maintained in this medium. Sperm suspensions preincubated for $2 \mathrm{~h}$ in glucose-containing medium yielded high fertilization levels with intact and zona-free eggs $(92.2$ and $100 \%$, respectively, Table 3 ), and terminal stages of egg activation and sperm head decondensation predominated. The only characteristic revealing significant differences between these 2 groups was the incidence of polyspermy which was much higher in the absence of zonae (Table 3 ).

Table 3. The effect of $5.56 \mathrm{mM}$-glucose (G) and/or $0.1 \mathrm{~mm}$-dibutyryl cyclic AMP (dbcAMP) on the ability of mouse spermatozoa to fertilize zona-intact and zona-free eggs

\begin{tabular}{|c|c|c|c|c|c|c|c|c|c|c|c|}
\hline \multicolumn{2}{|c|}{ Medium for } & \multirow[b]{2}{*}{ \pm Zona } & \multirow{2}{*}{$\begin{array}{l}\text { No. } \\
\text { of } \\
\text { exps }\end{array}$} & \multirow[b]{2}{*}{ Fertilized } & \multicolumn{3}{|c|}{ Egg stage, \%† } & \multicolumn{3}{|c|}{ Sperm stage, \%† } & \multirow{2}{*}{$\begin{array}{c}\text { Polyspermy, } \\
\% \\
(\% \\
\text { dispermic) }\end{array}$} \\
\hline Preincubation ${ }^{*}$ & Fertilization & & & & EA & M-LA & T-PB2 & 1 & 2 & 3 & \\
\hline \multirow[t]{2}{*}{ G } & G & + & 4 & $\begin{array}{c}47 / 51 \\
(92.2 \%)\end{array}$ & 0 & 0 & 100 & $2 \cdot 1$ & 0 & 97.9 & $4 \cdot 3 \ddagger(100)$ \\
\hline & & - & 4 & $\begin{array}{c}39 / 39 \\
(100 \%)\end{array}$ & 0 & 0 & 100 & 0 & 0 & 100 & $69 \cdot 2 \ddagger(55 \cdot 6)$ \\
\hline \multirow[t]{2}{*}{ dbcAMP } & dbcAMP & + & 4 & $\begin{array}{c}0 / 38 \\
(0 \%)\end{array}$ & - & - & - & - & - & - & - \\
\hline & & - & 5 & $\begin{array}{c}23 / 77 \\
(29.9 \%)\end{array}$ & 0 & $4 \cdot 4$ & 95.6 & 4.4 & 0 & $95 \cdot 6$ & $4 \cdot 3 \quad(100)$ \\
\hline dbcAMP & G & - & 2 & $\begin{array}{c}23 / 23 \\
(100 \%)\end{array}$ & 0 & 0 & 100 & 0 & 0 & 100 & $65.2 \quad(66 \cdot 7)$ \\
\hline
\end{tabular}

\footnotetext{
E, early anaphase; M-LA, middle-late anaphase; T-PB2, telophase-2nd polar body.

* For $2 \mathrm{~h}$.

+ Eggs were fixed after $75 \mathrm{~min}$.

$\ddagger$ Significantly, $P<0.001$.
}

Spermatozoa preincubated in medium containing dbcAMP only were completely unable to fertilize intact eggs, but fertilized about $30 \%$ of zona-free eggs; similar results (not shown) were obtained with sperm suspensions preincubated for $30 \mathrm{~min}$. Those eggs that were fertilized were apparently penetrated soon after mixing with spermatozoa because terminal nuclear states were observed in the majority; only 1 egg in this group was found to be polyspermic. In striking contrast, when these same sperm suspensions were diluted in glucose-containing medium immediately before eggs were introduced, all eggs were fertilized and possessed terminal stages of egg and sperm nuclear development; furthermore, the majority were polyspermic.

In all three groups, most of the polyspermic zona-free eggs were dispermic; in glucose-containing medium, approximately $25 \%$ were trispermic. Two eggs had asynchronously developing sperm heads with 1 out of 3 or 4 at an earlier stage than the others; all sperm heads except for these 2 were at stage 3 , i.e. fully decondensed. 


\section{Acrosome loss}

In all media tested, except that lacking both glucose and cyclic nucleotide, the proportion of motile spermatozoa that had undergone an acrosome reaction increased as the incubation period lengthened. In freshly prepared suspensions ( $5 \mathrm{~min}$ ), few cells had lost the acrosome, whether the medium contained glucose or cyclic nucleotide; similar low loss was observed in the glucose- and dbcAMP-free group at $2 \mathrm{~h}$. At $30 \mathrm{~min}$ and $2 \mathrm{~h}$ the results in the remaining media were similar and a progressive loss of acrosome was indicated (Table 4); at $2 \mathrm{~h}$ about $40 \%$ of spermatozoa in all media lacked an acrosome. There were therefore no significant differences for spermatozoa in 5 of the media tested, despite the considerable and striking differences in fertilizing ability (Table 1). Acrosome loss was detected in media which failed to promote whiplash motility (e.g. containing only cyclic nucleotide) as well as in glucose-containing media, indicating that the pattern of motility did not affect sperm passage down the gel column.

Table 4. Acrosome loss in mouse spermatozoa incubated in media containing $5.56 \mathrm{mM}$-glucose $(\mathrm{G})$ and/or dibutyryl cyclic AMP (dbcAMP)

\begin{tabular}{|c|c|c|c|c|}
\hline \multirow[b]{2}{*}{ Medium } & \multicolumn{4}{|c|}{ Acrosome loss (\%) during incubation for: } \\
\hline & $5 \mathrm{~min}$ & $30 \mathrm{~min}$ & $2 \mathrm{~h}$ & $4 \mathrm{~h}$ \\
\hline G & $6.9 \pm 0.4$ & $12 \cdot 4 \pm 5 \cdot 0$ & $37.7 \pm 3.0$ & $49.4 \pm 6.5$ \\
\hline $\mathrm{G}+0.1 \mathrm{mM}$-dbcAMP & -* & $16.7 \pm 0.8$ & $42 \cdot 8 \pm 2 \cdot 6$ & —* \\
\hline $0.1 \mathrm{mM}$-dbcAMP & $6 \cdot 5 \pm 1 \cdot 5$ & $18 \cdot 5 \pm 2.3$ & $42.4 \pm 5.4$ & $58 \cdot 1 \pm 8 \cdot 6$ \\
\hline $\mathrm{G}+1 \mathrm{mM}-\mathrm{dbcAMP}$ & 二* & $27 \cdot 1 \pm 8.9$ & $39.7 \pm 3.4$ & $-^{*}$ \\
\hline $1 \mathrm{mM}-\mathrm{dbc}$ AMP & -* & $21.7 \pm 7.0$ & $43.3 \pm 2.7$ & —* \\
\hline Glucose-free & 一* & —* & $7 \cdot 8 \pm 0.6$ & $-^{*}$ \\
\hline
\end{tabular}

Values are mean \pm s.d. Assessments were made after gel filtration to concentrate motile gametes.

* Not tested.

\section{Motility patterns}

In general, the proportion of motile gametes in all groups was similar. All media which contained glucose, irrespective of other components, or to which glucose was introduced just before assessment supported whiplash motility in $>50 \%$ of motile gametes after a 2 h incubation period. After $30 \mathrm{~min}$ in glucose-containing medium, very few $(<5 \%)$ of the gametes exhibited whiplash motility, although after an equivalent period in medium containing glucose $+1 \mathrm{~mm}$ - or 0.1 mM-dbcAMP, a significant proportion of spermatozoa (20-40\%) displayed whiplash motility. While media containing cyclic nucleotide but lacking glucose supported the acrosome reaction, no whiplash motility was observed in spermatozoa incubated for $30 \mathrm{~min}$ or $2 \mathrm{~h}$; rather, the characteristic, slightly sluggish motility exhibited in glucose-free medium (Fraser \& Quinn, 1981) was maintained throughout the incubation. The addition of glucose to these suspensions, however, was associated with an immediate transition to the whiplash pattern associated with fertilizing ability (Fraser, 1977).

\section{Discussion}

The results presented here suggest that capacitation of mouse spermatozoa involves either synthesis of cyclic AMP or maintenance of a critical level of this compound. By supplying exogenous cyclic nucleotide in the form of dibutyryl cyclic AMP as in the present experiments or by increasing endogenous levels through application of the phosphodiesterase inhibitor caffeine 
(Fraser, 1979b), the time required for capacitation is significantly decreased; when glucose is also present, spermatozoa treated in these ways for $30 \mathrm{~min}$ are able to penetrate eggs rapidly and synchronously, unlike spermatozoa maintained in medium containing only glucose. Furthermore, dibutyryl cyclic AMP supports the acrosome reaction in vitro, even in the absence of glucose (Table 4), whereas medium lacking both cyclic nucleotide and glucose fails to do so (Table 4; Fraser \& Quinn, 1981). Cyclic nucleotide alone, however, does not promote whiplash motility; in the absence of glucose, the occurrence of the acrosome reaction in isolation from motility changes will not permit fertilization of eggs with intact zonae, although some eggs can be fertilized after removal of this layer (Table 3).

These results suggest that the pivotal role played by glycolytic metabolism in the development of mouse sperm fertilizing ability is associated with the endogenous generation of cyclic AMP. That this generation may occur in the vicinity of the acrosome is consistent both with the demonstrated relationship between cyclic nucleotide and the acrosome reaction and with the localization of adenylate cyclase in sperm plasma membrane and outer acrosomal membrane fractions (Braun \& Dods, 1975; Herman, Zahler, Doak \& Campbell, 1976). The ATP required by adenylate cyclase for cyclic AMP synthesis may prove to be more accessible when supplied by the cytoplasmically-generated ATP from glycolysis than by the more remote mitochondrial oxidative metabolism occurring in the sperm midpiece. Cyclic nucleotide also plays an important role in development of the whiplash motility as indicated by the precocious expression of this motility in the presence of exogenously supplied dibutyryl cyclic AMP. Cyclic nucleotides, phosphodiesterase inhibitors and catecholamines, compounds which affect cyclic AMP levels in many systems examined, have been shown to stimulate glycolysis and/or motility in spermatozoa from many mammalian species (see 'Introduction'; Hoskins, 1973; Bavister, Chen \& Fu, 1979). Earlier studies had noted a general increase in glycolysis and respiration with capacitation (Hamner \& Williams, 1963; Mounib \& Chang, 1964), although the cause was not understood. If one assumes that ATP is the critical product of glycolysis, then this effect of cyclic nucleotide on motility may be due primarily to stimulation of glycolysis leading to increased availability of ATP, serving both for production of more cyclic AMP and as an energy substrate for motility. In many systems cyclic nucleotides exert their effect by activation of protein kinases; such kinases have been isolated from mammalian spermatozoa (Hoskins, Casillas \& Stephens, 1972; Garbers, First \& Lardy, 1973) and implicated in the generation of motility. Whatever the exact mechanism, cyclic AMP must act as an intermediary in this process since active glycolytic metabolism is also required to obtain the observed motility changes.

The vital role played by the whiplash motility in permitting mouse spermatozoa to traverse the zona pellucida is evident from the results presented in Table 3. It has generally been assumed that this vigorous tail movement is associated with zona penetration (Yanagimachi, 1970; Rogers \& Morton, 1973), but the present results unequivocally demonstrate the need for this characteristic form of movement. Only when the zona has been removed can sperm suspensions, capable of undergoing the acrosome reaction but lacking whiplash motility, fertilize eggs. Even then the relatively low success rate would suggest that motility may be crucial for proper orientation before and during sperm-egg fusion, and Wolf \& Armstrong (1978) have reported that motility is maintained during this phase of the fertilization process. However, by simply adding glucose to these same suspensions, a population which exhibits poor fertilizing capabilities is immediately transformed into a highly successful one, with the only major detectable change being the development of whiplash motility. In contrast to studies utilizing hamster sperm suspensions (Meizel, 1978), there was no evidence to suggest an association between mouse sperm motility pattern and loss of the acrosome (see Table 4); acrosome loss was similar in media containing cyclic nucleotide whether or not glucose was present to promote whiplash motility.

These results suggest that acrosome loss per se does not necessarily accurately reflect functional ability when spermatozoa are in the presence of eggs. The proportions of spermatozoa 
lacking acrosomes after a 30 min preincubation did not differ markedly whether medium contained only glucose or glucose plus $0.1 \mathrm{mM}$-dbcAMP, yet the latter group were functionally superior, being able to penetrate eggs in a synchronous manner soon after mixing of gametes. Negative data on mouse sperm acrosome loss are more relevant to fertilizing ability, i.e. the failure to achieve reasonable acrosome loss after a time sufficient to support capacitation (e.g. 2 h) is a reflection of functional shortcomings (Fraser \& Quinn, 1981). According to the generally accepted definition, capacitation is a process which prepares the spermatozoon to undergo the acrosome reaction (Bedford, 1970); it does not require overt acrosome loss in the absence of eggs, although clearly a certain proportion of cells will exhibit such loss after sufficient time in appropriate conditions.

Contrary to the report of Wolf, Inoue \& Stark (1976) that enzymic removal of the zona interfered with subsequent fertilization, no difficulties were encountered when a dialysed enzyme preparation promoting rapid zona dissolution was used. Removal of the zona leaves the egg plasma membrane immediately accessible to the spermatozoa and it is not surprising that the majority of zona-free eggs fertilized in glucose-containing medium were polyspermic. All but 2 possessed synchronously developing, fully decondensed sperm heads. These results argue strongly that the mouse egg block to polyspermy is efficient and rapid, probably occurring within a few minutes; this is consistent with the observations of Fukuda \& Chang (1978) and Sato (1979). Cytological (Fraser, 1979b) and chromosomal assessments (Fraser \& Maudlin, 1978) of zona-intact eggs indicate that the vast majority of multiple penetrations occur synchronously as demonstrated by the similarity of stages present, although the incidence of polyspermy is somewhat lower.

The observation that $1 \mathrm{~mm}$-dbcAMP interfered with fertilization, while $0.1 \mathrm{~mm}$ significantly accelerated capacitation, suggests that the inhibition of fertilization by guinea-pig spermatozoa obtained by Rogers \& Garcia (1979) with 10 mM-dbcAMP may be due to the high concentration tested. Alternatively, species differences may be such that critical cyclic nucleotides vary; Santos-Sacchi \& Gordon (1980) have reported that dibutyryl cyclic GMP, rather than AMP, can induce the acrosome reaction in guinea-pig spermatozoa. However, Mrsny \& Meizel (1980) found that micromolar amounts of dibutyryl cyclic AMP stimulated acrosome loss and motility changes in hamster spermatozoa, while the same compound at $10 \mathrm{~mm}$ had no detectable effect compared to nucleotide-free control samples. In the mouse system, manipulation of glycolytic metabolism by excluding glucose during the preincubation period effectively removed the inhibitory effect, suggesting that the inhibition could well be due to excess of a metabolite, such as butyrate, freed during metabolism of the dibutyryl analogue, this processing being dependent on glycolytic metabolism. The inhibition cannot be ascribed to a negative effect on the eggs since the concentration of cyclic nucleotide to which the eggs were exposed was the same, whether or not glucose was included during the sperm preincubation period (Table 2).

A caveat which follows from these particular experiments utilizing $1 \mathrm{~mm}$-dbcAMP is that, by assessment of acrosome loss and whiplash motility, the sperm suspensions would be expected to be highly fertile. After $2 \mathrm{~h}$ preincubation, approximately $40 \%$ of motile gametes had lost the acrosome and the majority exhibited whiplash motility, yet when mixed with eggs, fertilization was very poor. The ultimate proof of sperm capacitation still requires demonstration of functional ability within a strictly defined temporal framework rather than reliance solely on changes occurring in the absence of eggs.

Under standard conditions in the presence of glucose, mouse sperm suspensions require approximately $2 \mathrm{~h}$ to become fully capacitated, i.e. to fertilize a group of eggs rapidly and synchronously (Fraser \& Drury, 1976; Fraser, 1979b; Fraser \& Quinn, 1981). During that time, $35-40 \%$ of motile cells lose the acrosome and the majority develop whiplash motility (Fraser, 1977; Fraser \& Quinn, 1981; present study). In the absence of glycolytic metabolism, neither change is observed, but the addition of glucose at the time eggs are introduced permits an immediate shift to the levels observed in the former group and fertilization occurs rapidly and 
synchronously. In the presence of dibutyryl cyclic AMP, capacitation time is shortened as indicated by rapid and synchronous fertilization after a 30 -min preincubation rather than the usual one of $2 \mathrm{~h}$. These results strongly suggest that the process of capacitation in mouse spermatozoa involves the endogenous generation of cyclic AMP, with the essential requirements for cyclic nucleotide synthesis, the acrosome reaction and whiplash motility being supplied by glycolytic metabolism. This proposed role for cyclic AMP is consistent with several recent studies, including the observed correlation between an endogenous rise in cyclic AMP levels and induction of the acrosome reaction in guinea-pig spermatozoa (Hyne \& Garbers, 1979) and a stimulation of motility, acrosome loss and fertilizing ability in hamster spermatozoa by catecholamines (Cornett \& Meizel, 1978; Cornett, Bavister \& Meizel, 1979). One temporal factor underlying capacitation may therefore prove to be either activation of adenylate cyclase or inactivation of phosphodiesterases or a combination of both processes. When exogenous cyclic nucleotide is supplied, as in the present experiments, the lag needed for such activation is circumvented, capacitation time is shortened and spermatozoa can fertilize rapidly. Reliance on endogenous cyclic nucleotide production through changes in enzyme activity necessitates a longer time before spermatozoa can fertilize eggs, thus accounting for the patterns of fertilization obtained in cyclic nucleotide-free medium.

I thank Dr Peter J. Quinn for many stimulating discussions and for suggesting the possible inhibitory affect of butyrate on sperm fertilizing ability.

\section{References}

Bavister, B.D., Chen, A.F. \& Fu, P.C. (1979) Catecholamine requirement for hamster sperm motility in vitro. J. Reprod. Fert. 56, 507-513.

Bedford, J.M. (1970) Sperm capacitation and fertilization in mammals. Biol. Reprod., Suppl. 2, 128-158.

Braun, T. \& Dods, R.F. (1975) Development of a $\mathrm{Mn}^{2+}$-sensitive, "soluble" adenylate cyclase in rat testis. Proc. natn. Acad. Sci. U.S.A. 72, 1097-1101.

Cornett, L.E. \& Meizel, S. (1978) Stimulation of in vitro activation and acrosome reaction of hamster sperm by catecholamines. Proc. natn. Acad. Sci. U.S.A. 75, 4954-4958.

Cornett, L.E., Bavister, B.D. \& Meizel, S. (1979) Adrenergic stimulation of the fertilizing activity in hamster spermatozoa. Biol. Reprod. 20, 925-929.

Fraser, L.R. (1977) Motility patterns in mouse spermatozoa before and after capacitation. J. exp. Zool. 202, 439-444.

Fraser, L.R. (1979a) Rate of fertilization in vitro and subsequent nuclear development as a function of the post-ovulatory age of the mouse egg. J. Reprod. Fert. 55, 153-160.

Fraser, L.R. (1979b) Accelerated mouse sperm penetration in vitro in the presence of caffeine. $J$. Reprod. Fert. 57, 377-384.

Fraser, L.R. \& Drury, L.M. (1975) The relationship between sperm concentration and fertilization in vitro of mouse eggs. Biol. Reprod. 13. 513-518.

Fraser, L.R. \& Drury, L.M. (1976) Mouse sperm genotype and the rate of egg penetration in vitro. $J$. exp. Zool. 197, 13-20.

Fraser, L.R. \& Maudlin, I. (1978) Relationship between sperm concentration and incidence of polyspermy in mouse embryos fertilized in vitro.J. Reprod. Fert.52, 103-106.
Fraser, L.R. \& Quinn, P.J. (1981) A glycolytic product is obligatory for initiation of the sperm acrosome reaction and whiplash motility required for fertilization in the mouse. J. Reprod. Fert. 61, 25-35.

Fukuda, Y. \& Chang, M.C. (1978) The time of cortical granule breakdown and sperm penetration in mouse and hamster eggs inseminated in vitro. Biol. Reprod. $19,261-266$.

Garbers, D.L., First, N.L., Sullivan, J.J. \& Lardy, H.A. (1971) Stimulation and maintenance of ejaculated bovine spermatozoan respiration and motility by caffeine. Biol. Reprod. 5, 336-339.

Garbers, D.L., Lust, W.D., First, N.L. \& Lardy, H.A. (1971) Effects of phosphodiesterase inhibitors and cyclic nucleotides on sperm respiration and motility. Biochemistry, N.Y. 10, 1825-1831.

Garbers, D.L., First, N.L. \& Lardy, H.A. (1973) Properties of adenosine $3^{\prime}, 5^{\prime}$-monophosphatedependent protein kinases isolated from bovine epididymal spermatozoa. J. biol. Chem. 248, 875879.

Hamner, C.R. \& Williams, W.L. (1963) Effect of the female reproductive tract on sperm metabolism in the rabbit and fowl. J. Reprod. Fert. 5, 143-150.

Herman, C.A., Zahler, W.L., Doak, G.A. \& Campbell, B.J. (1976) Bull sperm adenyl cyclase: localization and partial characterization. Arch. Biochem. Biophys. 177, 622-629.

Hicks, J.J., Martinez-Manautou, J., Pedron, N. \& Rosado, A. (1972) Metabolic changes in human spermatozoa related to capacitation. Fert. Steril. 23, 172-179.

Hoskins, D.D. (1973) Adenine nucleotide mediation of fructolysis and motility in bovine epididymal spermatozoa. J. biol. Chem. 248, 1135-1140. 
Hoskins, D.D., Casillas, E.R. \& Stephens, D.T. (1972) Cyclic AMP-dependent protein kinases of bovine epididymal spermatozoa. Biochem. Biophys. Res. Commun. 48, 1331-1338.

Hyne, R.V. \& Garbers, D.L. (1979) Calcium-dependent increase in adenosine $3^{\prime}, 5^{\prime}$-monophosphate and induction of the acrosome reaction in guinea pig spermatozoa. Proc. natn. Acad. Sci. U.S.A. 76, 5699-5703.

Meizel, S. (1978) The mammalian sperm acrosome reaction, a biochemical approach. In Development in Mammals, vol. 3, pp. 1-64. Ed. M. H. Johnson. Elsevier-North Holland, Amsterdam.

Morton, D.B. (1976) Lysosomal enzymes in mammalian spermatozoa. In Lysosomes in Biology and Pathology, Vol. 5, pp. 203-255. Eds J. T. Dingle \& R. T. Dean. Elsevier-North Holland, Amsterdam.

Mounib, M.S. \& Chang, M.C. (1964) Effect of in utero incubation on the metabolism of rabbit spermatozoa. Nature, Lond. 201, 943-944.

Mrsny, R.J. \& Meizel, S. (1980) Evidence suggesting a role for cyclic nucleotides in acrosome reactions of hamster sperm in vitro. J. exp. Zool. 211, 153-157.

Rogers, B.J. \& Garcia, L. (1979) Effect of cAMP on acrosome reaction and fertilization. Biol. Reprod. 21, 365-372.

Rogers, B.J. \& Morton, B. (1973) ATP levels in hamster spermatozoa during capacitation in vitro. Biol. Reprod. 9, 361-369.

Rosado, A., Hicks, J.J., Reyes, A. \& Blanco, I. (1974) Capacitation in vitro of rabbit spermatozoa with cyclic adenosine monophosphate and human follicular fluid. Fert. Steril. 25, 821-824.

Santos-Sacchi, J. \& Gordon, M. (1980) Induction of the acrosome reaction in guinea pig spermatozoa by cGMP analogues. J. Cell Biol. 85, 798-803.

Sato, K. (1979) Polyspermy-preventing mechanisms in mouse eggs fertilized in vitro. J. exp. Zool. $\mathbf{2 1 0}$, $353-360$.

Schoenfeld, C., Amelar, R.D. \& Dubin, L. (1975) Stimulation of ejaculated human spermatozoa by caffeine. Fert. Steril. 26, 158-161.

Toyoda, Y. \& Chang, M.C. (1974) Capacitation of epididymal spermatozoa with high $\mathrm{K} / \mathrm{Na}$ ratio and cyclic AMP for the fertilization of rat eggs in vitro. $J$. Reprod. Fert. 36, 125-134.

Wolf, D.P. \& Armstrong, P.B. (1978) Penetration of the zona-free mouse egg by capacitated epididymal sperm: cinemicrographic observations. Gamete Res. $1,39-46$.

Wolf, D.P., Inoue, M. \& Stark, R.A. (1976) Penetration of zona-free mouse eggs. Biol. Reprod. 15, 213-221.

Yanagimachi, R. (1970) The movement of golden hamster spermatozoa before and after capacitation. J. Reprod. Fert. 23, 193-196.

Yanagimachi, R. \& Usui, N. (1974) Calcium dependence of the acrosome reaction and activation of guinea pig spermatozoa. Expl Cell Res. 89, 161-174.

Received 16 June 1980 